the association is explained by smoking, chronic bronchitis or allergy as these conditions commonly occur in asthma individuals. The aim of this prospective cohort study was therefore to explore the association between asthma, levels of asthma control and lung cancer incidence, taking into account the commonly occurring conditions.

Methods We followed 63,103 adults who participated in the second survey of the HUNT Study in Norway from 1995-97 to 2017. None of the participants had known cancer at the time of inclusion. Ever asthma (9.0\%), doctor-diagnosed asthma (5.5\%) and doctor-diagnosed active asthma (3.7\%) were defined based on self-reported information at baseline. Among individuals with doctor-diagnosed active asthma, levels of asthma control were categorized into well controlled and poorly controlled. Incident lung cancer cases were ascertained from the Cancer Registry of Norway. Cox regression models were used to estimate adjusted hazard ratios (HRs) with 95\% confidence intervals (CIs) for incident lung cancer in relation to asthma status.

Results In total, 1,013 participants developed lung cancer during a median follow-up of 21.1 years. After adjustment for smoking (classified into detailed categories based on information of smoking status and pack-years), chronic bronchitis, allergy and other confounders, increased overall incidence of lung cancer was associated with ever asthma (HR 1.32, 95\% CI 1.09-1.61), doctor-diagnosed asthma (HR 1.32, 95\% CI 1.04-1.67) and doctor-diagnosed active asthma (HR 1.40, 95\% CI 1.08-1.82). Individuals with ever asthma only and without current smoking, chronic bronchitis or allergy appeared to have an increased incidence of lung cancer compared with those with no ever asthma and no such common condition. Poorly controlled doctor-diagnosed active asthma was associated with an increased incidence of lung cancer (HR 1.57, 95\% CI 1.14-2.16), whereas no clear association between well-controlled doctor-diagnosed active asthma and lung cancer was observed (HR 1.16, 95\% CI 0.65-2.06).

Conclusion Our study suggested that asthma, in particular poorly controlled asthma, was associated with an increased lung cancer incidence. Smoking, chronic bronchitis and allergy did not seem to explain the association.

\section{OP04 TIME-TRENDS IN INCIDENCE OF GASTRIC CANCER BY SITE AND HISTOTYPE IN THE COMMUNITY IN ITALY}

\footnotetext{
1,2WJ Harrison* ${ }^{3}{ }^{3} \mathrm{~A}$ Romiti, ${ }^{2} \mathrm{GR}$ Law, ${ }^{3} \mathrm{~F}$ Bazzoli, ${ }^{3} \mathrm{RM}$ Zagari. ${ }^{1}$ Leeds Institute for Data Analytics, University of Leeds, Leeds, UK; ${ }^{2}$ School of Medicine, University of Leeds, Leeds, UK; ${ }^{3}$ Department of Medical and Surgical Sciences, University of Bologna, Bologna, Italy
}

\subsection{6/jech-2019-SSMabstracts.4}

Background The incidence of gastric cancer is decreasing worldwide, but little is known about how the incidence of different types of gastric adenocarcinoma (GAC), such as noncardia and cardia subsites, or intestinal and diffuse histotypes, have been changing over time. This study explores the incidence of GAC stratified by anatomic site and histotype in the community in Italy.

Methods A multicentre, retrospective, observational study was performed to identify all cases of GAC diagnosed in the community of Bologna and province over a twelve year period from 2001 to 2012. All histological reports of new cases of GAC diagnosed in the study period in the four hospitals of Bologna and province were identified. First histological diagnoses only were included, based on endoscopic biopsies or surgical specimens. Patients resident outside of the region were excluded. The anatomic site (non-cardia or cardia) and the histotype (intestinal or diffuse) were identified. Directly age-standardised incidence rates per year per 100,000 adults were calculated for males and females using the European standard population.

Results A total of 2,895 cases of GAC were identified. The majority were non-cardia $(2,510 ; 87 \%)$, and were either intestinal $(1,409 ; 49 \%)$ or diffuse $(972 ; 34 \%)$ histotypes. More cases were seen in males $(1,673 ; 58 \%)$ than in females $(1,222 ; 42 \%)$. For all GAC, incidence rates decreased from 2001 to 2012 in both males [from 50.0 (95\% CI 42.6-57.3) to 28.8 (95\% CI 23.5-34.0)] and females [from $24.3(95 \%$ CI 19.9-28.7) to 16.6 (95\% CI 13.1-20.1)]. A similar pattern was seen for non-cardia GAC, but not for cardia GAC, whose incidence rates remained stable over time. Incidence rates of intestinal type GAC decreased substantially in males [from 26.8 (95\% CI 21.4-32.2) to 16.2 (95\% CI 12.3-20.1)] and, to a lesser extent, in females [from 9.1 (95\% CI 6.5-11.8) to 5.2 (95\% CI 3.3-7.1)]. Incidence rates of diffuse type GAC also decreased in males [from 14.8 (95\% CI 10.7-18.8) to 6.9 (95\% CI 4.4-9.5)], but less so in females [from 10.4 (95\% CI 7.3-13.4) to 8.1 (95\% CI 5.6-10.7)].

Conclusion Incidence rates of GAC are decreasing over time in this community in Italy for both males and females. The decline seems to be limited to non-cardia GAC, the intestinal histotype and predominantly to males for the diffuse histotype. Unmeasured risk factors such as $H$. pylori infection and diet may contribute to the differences. These data yield important information to aid healthcare planning in the region.

\section{OP05 SIMULATION OF THE IMPACT OF TOBACCO CONTROL POLICIES ON FUTURE CANCER INCIDENCE IN GERMANY (2020-2050)}

1,2T Gredner*, ${ }^{1} \mathrm{~T}$ Niedermaier, ${ }^{1,3,4} \mathrm{H}$ Brenner, ${ }^{1,5} \mathrm{U}$ Mons. ${ }^{1}$ Division of Clinical Epidemiology and Aging Research, German Cancer Research Center (DKFZ), Heidelberg, Germany; ${ }^{2}$ Medical Faculty Heidelberg, University of Heidelberg, Heidelberg, Germany; ${ }^{3}$ Division of Preventive Oncology, German Cancer Research Center (DKFZ), Heidelberg, Germany; ${ }^{4}$ German Cancer Consortium (DKTK), German Cancer Research Center (DKFZ), Heidelberg, Germany; ${ }^{5}$ Cancer Prevention Unit, German Cancer Research Center (DKFZ), Heidelberg, Germany

\subsection{6/jech-2019-SSMabstracts.5}

Background Despite reductions in smoking prevalence over the past decades, smoking remains the most important preventable cancer risk factor in Germany. In contrast to a considerable attributable disease burden, Germany continues to be ranked among the most inactive countries in Europe in terms of implementing evidence-based tobacco control policies. The aim of this study is to provide projections of potentially avoidable cancer cases under different policy intervention scenarios.

Methods In order to estimate the proportion of potentially avoidable cancer cases under different policy intervention scenarios (tobacco tax increases, comprehensive marketing ban, plain packaging), we calculated cancer site-specific potential impact fractions (PIFs) stratified according to age and sex, for each year of study period (2020-2050), considering latency periods between the reduction in smoking prevalence and the manifestation in declining cancer excess risks. For the baseline scenario we assumed a continuation of recent smoking trends, and combined data of the German cancer registries with forecasted population sizes, published effect sizes, and national 